\title{
Local crystallographic texture and voiding in passivated copper interconnects
}

\author{
J. A. Nuccia) \\ School of Electrical Engineering, Phillips Hall, Cornell University, Ithaca, New York 14853 \\ R. R. Keller \\ National Institute of Standards and Technology, Materials Reliability Division, Boulder, Colorado 80303 \\ J. E. Sanchez, Jr. \\ Department of Materials Science and Engineering, 2122 Dow Building, University of Michigan, \\ Ann Arbor, Michigan 48109 \\ Y. Shacham-Diamand \\ School of Electrical Engineering, Phillips Hall, Cornell University, Ithaca, New York 14853
}

(Received 9 May 1996; accepted for publication 24 October 1996)

\begin{abstract}
A correlation between local crystallographic texture and stress-induced void formation in tantalum-encapsulated, copper interconnects was revealed by electron backscattering diffraction studies in a scanning electron microscope. Lines exhibiting an overall stronger $\langle 111\rangle$ texture showed better resistance to void formation. Furthermore, grains adjacent to voids exhibited weaker $\langle 111\rangle$ texture than grains in unvoided regions of the same line. The locally weaker $\langle 111\rangle$ texture at voided locations suggests the presence of higher diffusivity, twist boundaries. This work, which represents the first characterization of local texture in stress voided, copper lines, helps to elucidate the relative importance of the thermodynamic and kinetic factors which govern void formation and growth. (c) 1996 American Institute of Physics. [S0003-6951(96)04252-0]
\end{abstract}

The reliability of advanced integrated circuit devices is limited largely by interconnect failures. Aspects of aluminum film microstructure, including the grain size, ${ }^{1}$ grain size distribution, ${ }^{2}$ and crystallographic texture ${ }^{3}$ are known to correlate with electromigration and stress-induced voiding in patterned interconnects produced from blanket films. However, it has been shown that interconnect line microstructure may evolve differently from film microstructure. ${ }^{4,5}$ One study revealed that the $\langle 111\rangle$ texture of aluminum lines was stronger than that of blanket films. ${ }^{4}$ Such work reveals the importance of characterizing line microstructure.

Microstructural variations within a line must also be examined, in addition to understanding differences between blanket film and line microstructure. There is serious concern for the reliability of future generation devices since a detailed understanding of the local processes which produce failure is lacking. The local line microstructure at voided and/or failed sites must be compared to the microstructure associated with intact regions of the line to develop a better mechanistic understanding of reliability failure modes. Characterization of local microstructure at electromigrationinduced transgranular slit voids in aluminum alloy lines ${ }^{6}$ by transmission electron microscopy (TEM) ${ }^{7}$ and electron backscattering diffraction (EBSD) ${ }^{4,8}$ revealed typical $\langle 111\rangle$ fiberoriented grains at voided sites. While recent work has provided information regarding certain Al-based microstructures associated with stress-induced voiding, the results have been inconsistent. Kordic ${ }^{9}$ correlated decreased stress-induced void formation with stronger average $\langle 111\rangle$ line textures in $\mathrm{Al}-(\mathrm{Si}, \mathrm{Cu})$ alloys, however no local microstructural information at voided sites was reported. In contrast, stronger

a)Electronic mail: nucci@cnf.cornell.edu average $\langle 111\rangle$ texture was recently correlated with significantly increased stress-voiding in aluminum interconnects, ${ }^{10}$ with the further result that the grains at voided sites were significantly off- $\langle 111\rangle$ in orientation. Additional studies are needed to clarify the effect of microstructure on stressinduced voiding in aluminum lines.

If aluminum-based interconnects cannot provide the required reliability, future devices may require novel interconnect materials such as copper. Stress-induced voiding in passivated copper interconnects was recently examined as a function of linewidth and heat treatment. ${ }^{11}$ Copper lines $0.75-2.0 \mu \mathrm{m}$ wide and $0.5 \mu \mathrm{m}$ thick were electron-beam evaporated and patterned using a trilayer lift-off stack onto tantalum coated, thermally oxidized silicon wafers. The copper was cladded with $50 \mathrm{~nm}$ of sputtered tantalum and passivated with $1.2 \mu \mathrm{m}$ plasma-enhanced chemical vapor deposited oxide at $240^{\circ} \mathrm{C}$. The tantalum acted as both a diffusion barrier and an adhesion layer. Furthermore, tantalum has been shown to induce in copper a moderately strong $\langle 111\rangle$ texture, which improves thermal cycling behavior. ${ }^{12}$

Two heat treatments were employed to alter the line microstructure. Type 1 samples were annealed for $1 \mathrm{~h}$ at $400{ }^{\circ} \mathrm{C}$, only after passivation deposition (post-passivation anneal). Type 2 samples were annealed for $1 \mathrm{~h}$ at $450{ }^{\circ} \mathrm{C}$ immediately following copper lift-off and were subjected to the post-passivation anneal. All anneals were run in vacuum at a base pressure of $1 \times 10^{-5} \mathrm{~Pa}\left(2 \times 10^{-7}\right.$ Torr $)$ and concluded with a furnace cool to room temperature. The samples came from a single wafer to assure uniformity. Measurements from focused ion beam images revealed monomodal grain size distributions for each sample type, and average grain diameters of $0.53 \mu \mathrm{m}$ for type 1 and $0.56 \mu \mathrm{m}$ for type 2 samples, after the post-passivation anneals. Given the uncertainty in the grain size determination, the grain sizes are 
considered equivalent. Statistics were gathered for voids located at the line edge (edge voids) and within the line (central voids). For lines greater than $1 \mu \mathrm{m}$ wide, the central void population was directly proportional to the line area for both sample types. This dependence was linked to the number of grain boundary triple points available for void nucleation. As a result of a transition to bamboo microstructure for linewidths less than $1 \mu \mathrm{m}$, the number of triple points per unit area was no longer constant. That type 2 samples voided substantially less than the type 1 samples was less easily explained, however. The earlier study ${ }^{11}$ showed approximately a 4.5 times lower population of central voids for the type 2 samples than the type 1 samples.

A crystallographic analysis was subsequently conducted on the specimens described in Ref. 11 to elucidate the effect of heat treatment on voiding. Specifically, the local textures of these samples were examined using EBSD in the scanning electron microscope. ${ }^{13}$ EBSD evaluation enabled acquisition of a statistically significant data set with minimal sample preparation. Also, since the spatial resolution of the technique is approximately $0.2 \mu \mathrm{m}$, individual grains were analyzed and the orientations of grains at voided and unvoided locations were compared. Details of the EBSD setup are found elsewhere. ${ }^{14}$ The crystallographic orientations of grains adjacent to ten central and nine edge voids were analyzed. These voids were equally distributed between the type 1 and type 2 samples, in lines ranging from 0.75 to $2.0 \mu \mathrm{m}$ wide. Approximately three to four diffraction patterns were collected around edge voids and six to eight diffraction patterns were collected around central voids. For the present work, there was no distinction between data from edge voids and that from central voids. Each pattern was collected within approximately one grain diameter of a void. For comparison, additional EBSD patterns were acquired from arrays of beam positions in unvoided regions of the lines. The unvoided data sampled several micrometers along the line length and the entire line width for 1 and $2 \mu \mathrm{m}$ wide lines. For each heat treatment type, roughly 45 diffraction patterns adjacent to voids and 65 diffraction patterns within unvoided regions were analyzed.

The EBSD data were quantitatively evaluated in terms of pole plots, which show the $\langle 111\rangle$ orientation density in units of times random as a function of tilt angle away from the specimen normal. These are texture representations comparable to $x$-ray diffraction rocking curves and were obtained by integrating about the azimuthal angle of $\langle 111\rangle$ pole figures. ${ }^{15}$ Figure 1 shows a comparison between the overall line textures for the different sample types. The more void resistant, type 2 samples display an approximately $33 \%$ stronger $\langle 111\rangle$ texture than do the type 1 samples, as indicated by the maximum $\langle 111\rangle$ orientation density at $0^{\circ}$. A simplified analysis of the voided data is presented by summarizing all grain orientations measured around voids into a single texture plot. In this manner the overall, average texture of grains adjacent to voids can be visualized relative to that of unvoided regions. Figure 2 shows such a plot for the local texture at voided and unvoided regions within type 2 lines. Grains adjacent to voids show an approximately $35 \%$ weaker local $\langle 111\rangle$ texture than those in unvoided regions.

Inferences can now be made regarding the average grain

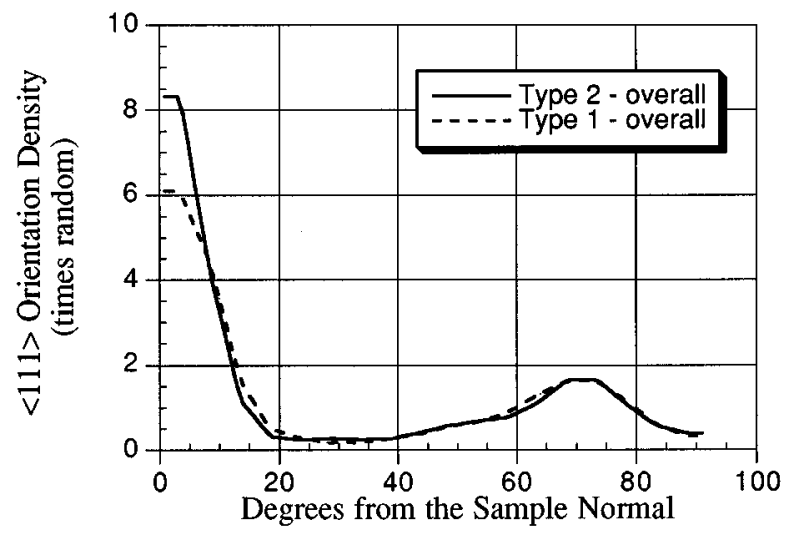

FIG. 1. Pole plots containing combined data from both unvoided and voided regions in type 1 and 2 samples illustrate the strengthening of $\langle 111\rangle$ fiber texture with heat treatment.

boundary character in type 1 and type 2 samples, as well as in voided regions within a line. A weaker texture implies the presence of a wider variation in grain boundary structures and properties. ${ }^{16}$ A general mixture of tilt and twist boundary structures is associated with a weaker $\langle 111\rangle$ texture. Conversely, stronger texture implies that a larger proportion of boundaries is of either pure tilt character or tilt with a low angle twist component. For the case of a tilt boundary with tilt axis normal to the film surface, pipe diffusion along edge dislocations parallel to the tilt axis is preferred. ${ }^{17,18}$ Thus, there is reduced average diffusion in tilt grain boundaries along directions parallel to the film surface. In contrast, diffusion within a twist boundary, which is typically composed of a network of screw dislocations, exhibits far less anisotropy and greater atomic mobility in the plane of the film. It is reasonable to assume that a twist boundary is capable of transporting matter further away form a growing void than a tilt boundary. For a given number of nucleation sites, faster void growth is expected in the twist case. These local measurements help to confirm the present and previous hypotheses made regarding grain boundary character and void resistance in highly textures $\langle 111\rangle$ films by identifying failures at regions of locally reduced $\langle 111\rangle$ texture. Improved electromigration resistance in strongly textured aluminum films was similarly explained in an earlier work. ${ }^{1}$

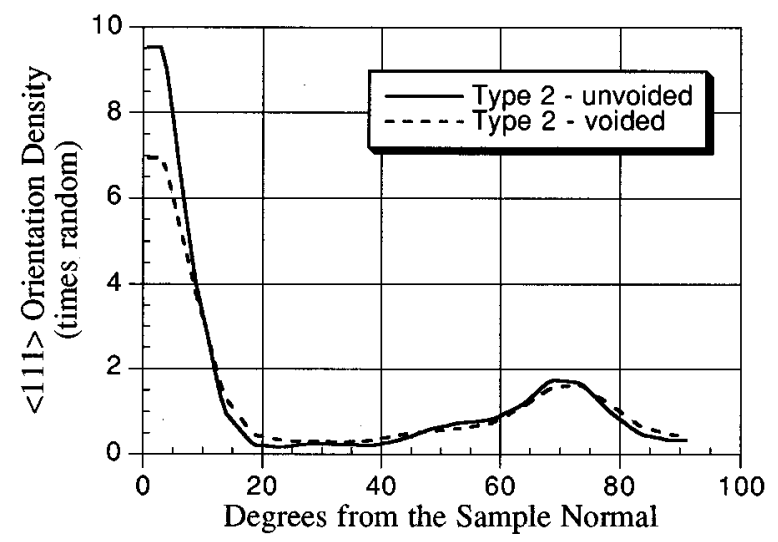

FIG. 2. Pole plots from type 2 samples reveal stronger $\langle 111\rangle$ fiber texture at unvoided line segments than adjacent to voids. This trend was also observed for type 1 samples. 
In addition to differences in the tilt/twist character of grain boundaries, differences in grain boundary misorientation angle may also affect voiding propensity. Perhaps the stronger texture in type 2 samples favors the formation of low angle grain boundaries. The work of Wardle et al. ${ }^{19}$ showed a relation between grain boundary misorientation angle and grain boundary diffusivity. Diffusivities increase as misiorientation angle increases, at least for low-angle grain boundaries. Measurements of grain boundary misorientation are underway in an effort to experimentally verify these anticipated changes in grain boundary character with film texture.

These experiments help to elucidate the relative importance of the driving forces versus the kinetics which control stress void formation. The differently heat treated samples had identical geometries and approximately equivalent grain sizes and distributions. As a result, they exhibited presumably identical average stress states and contained nearly the same number of void nucleation sites. While the reduction of stress is the thermodynamic driving force for voiding, this study suggests that the structure of the kinetic pathways greatly influences stress voiding reliability.

This work was supported by Semiconductor Research Corporation Contract No. 95-SC-069 and by the NIST Office of Microelectronic Programs. Samples were fabricated at the Cornell Nanofabrication Facility and analyzed at the Materials Science Center at Cornell University. Collaboration with David Field is graciously acknowledged. Thanks to Karin
Borgersen for assisting in the collection of void statistics and EBSD patterns, and to Xochitl Zamora-Thompson for assisting in pattern indexing.

${ }^{1}$ M. J. Attardo and R. Rosenberg, J. Appl Phys. 41, 2381 (1970).

${ }^{2}$ M. J. Attardo, R. Rutledge, and R. C. Jack, J. Appl. Phys. 42, 4343 (1971).

${ }^{3}$ S. Vaidya and A. K. Sinha, Thin Solid Films 75, 253 (1981).

${ }^{4}$ J. L. Hurd, K. P. Rodbell, D. B. Knorr, and N. L. Koligman, Mat. Res. Symp. Proc. 343, 653 (1994).

${ }^{5}$ D. P. Field and D. J. Dingley, J. Electron. Mater. 25, 1767 (1996).

${ }^{6}$ J. E. Sanchez, Jr., O. Kraft, and E. Artz, Appl. Phys. Lett. 61, 3121 (1992).

${ }^{7}$ J. H. Rose, Appl. Phys. Lett. 61, 2170 (1992).

${ }^{8}$ J. E. Sanchez, Jr., V. Randle, O. Kraft, and E. Arzt, Proc. SPIE 1805, 222 (1992).

${ }^{9}$ S. Kordic, R. A. M. Wolters, and K. Z. Troost, J. Appl. Phys. 74, 5391 (1993).

${ }^{10}$ K. P. Rodbell, J. L. Hurd, and P. W. DeHaven, Mat. Res. Soc. Proc. 428, (in press).

${ }^{11}$ J. A. Nucci, Y. Shacham-Diamand, and J. E. Sanchez, Jr., Appl. Phys. Lett. 66, 3585 (1995).

${ }^{12}$ R. P. Vinci and J. C. Bravman, Mat. Res. Soc. Symp. Proc. 309, 269 (1993).

${ }^{13}$ D. J. Dingley and V. Randle, J. Mater. Sci. 27, 4545 (1992),

${ }^{14}$ J. A. Nucci, doctoral dissertation, Cornell University, 1996.

${ }^{15}$ D. P. Field, P. R. Besser, J. E. Sanchez, Jr., and D. J. Dingley (unpublished).

${ }^{16}$ D. B. Knorr, D. P. Tracy, and K. P. Rodbell, Appl. Phys. Lett. 59, 3241 (1991).

${ }^{17}$ P. H. Pumphrey, Grain Boundary Structure and Properties, edited by G. A. Chadwick and D. A. Smith (Academic, New York, 1976), p. 139.

${ }^{18}$ N. L. Peterson, Grain Boundary Structure and Kinetics (ASM, Metals Park, OH, 1980), p. 209.

${ }^{19}$ S. C. Wardle, B. L. Adams, C. S. Nichols, and D. A. Smith, Mat. Res. Soc. Symp. Proc. 343, 666 (1994). 